\title{
Effect of the Changes in Bulk Density and Granulometric Properties on the Strength Properties of the Moulding Sand Mixtures
}

\author{
H. HUDÁK ${ }^{1}$, L. VARGA ${ }^{2}$ \\ ${ }^{1}$ University of Miskolc, Faculty of Materials Science and Engineering, Institute of Foundry, ontheni@uni- \\ miskolc.hu \\ ${ }^{2}$ University of Miskolc, Faculty of Materials Science and Engineering, Institute of Foundry, ontvlaci@uni- \\ miskolc.hu
}

Abstract. Foundry technology uses a lot of several natural materials. Sands use for preparing mixtures whereby making moulds or cores. Sand is defined as a granular, refractory major portion of mixture (90 - 98\% in dependence on used binder). Sand properties depend on it has chemical and mineralogical composition; mainly particle-size distribution and shape of grains and its size and sand surface texture. A comparative measurement of two quartz sand with different surface quality was carried out. Greensand mixtures were prepared to measure their permeability, compressive strength and wet tensile strength. The strength of sand mixtures has two main components. One of them is the cohesion of the binder; the other one is the adhesion between the binder and the foundry sand. The aim of this research is to determine the ratio of cohesion and adhesion within the strength values.

\section{Introduction}

The foundry moulds and sand cores are generally composed of 3 main components, which are: refractory, particulate material, which is always sand; binder, that holds together the sand particles, this can be organic or inorganic bonded; additive, which makes more preferential properties. Quartz sand is the most frequently applied type of foundry sands. Quartz sand is found in the nature in varying purity and is traded in varying quality for different industrial applications. The quality of castings produced depends largely upon the properties of the quartz sand utilized. A foundry mould must have the ability to withstand the high temperature of molten metal without damaging the contact surface between metal and sand. To ensure high-quality castings the quartz sand must satisfy specifications such as permeability, grain fineness, moisture, bond strength and refractoriness. Smooth-surfaced and rounded sands are more suitable for binders because sand has small surface therethrough minimal binder consumption needed. The quality of quartz sand determines the physical and chemical properties (e.g. grain size, shape, grain-size distribution, chemical composition, refractoriness). Based on the source materials, the sand core test specimens made of different surface quartz sands show even a double difference in their bending stress values [1-4].

Sands of almost the same granulometric but different micro surface properties can show variable behaviour in respect of the foundry use. The microsurface of the sands plays a very important role in 
the requirement for binders. Saving in the binder can be reached - achieving, however, the same strength, when choosing smooth surface sand [5].

\section{Materials}

A comparative measurement of two quartz sand with different surface quality was carried out. Quartz sand of two different surfaces was used for our tests (GBM 45 and SH 33). Figure 1. shows the grain morphology of different sands while a grain size distribution of them can be seen in Figure 2.

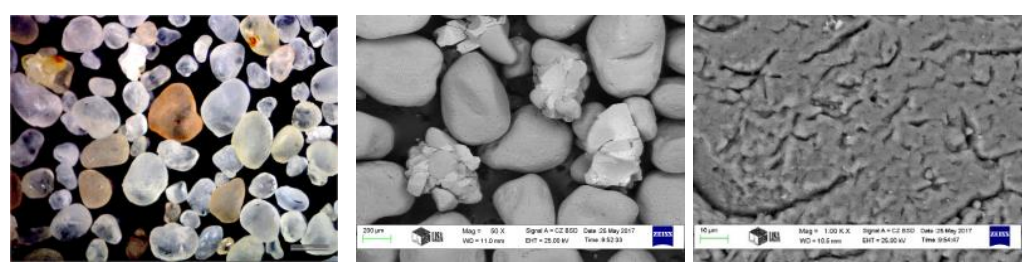

a)
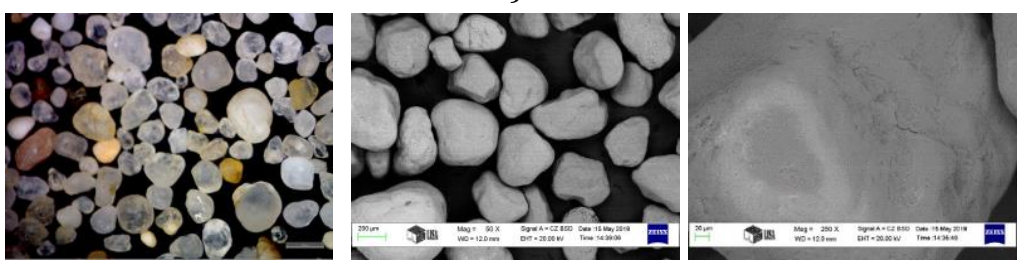

b)

Figure 1. Stereo and scanning electron microscopy (SEM) image of foundry sands.

a) $\quad \operatorname{GBM} 45(N=25 x),(N=50 x)$ and $(N=1000 x)$.

b) $\quad S H 33(N=25 x),(N=50 x)$ and $(N=250 x)$.
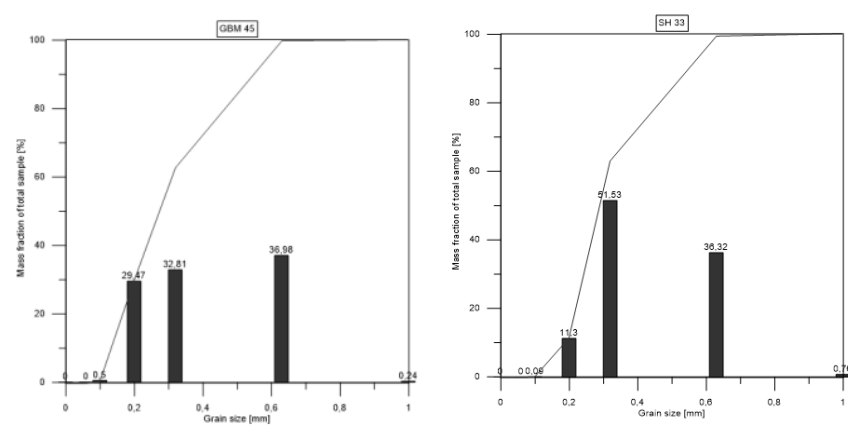

Figure 2. The grain size distribution of the used quartz sands.

Table 1. shows the measurement data of the investigated sands.

\begin{tabular}{|l|c|c|}
\hline & GBM 45 & $\boldsymbol{S H ~ 3 3 ~}$ \\
\hline Average granularity $\left(\mathrm{d}_{50}\right)$ & $0.303 \mathrm{~mm}$ & $0.333 \mathrm{~mm}$ \\
\cline { 2 - 3 } AFS granularity number & 61.7 & 51.9 \\
\cline { 2 - 3 } Homogeneity degree & $44 \%$ & $58 \%$ \\
\cline { 2 - 3 } $\begin{array}{l}\text { Angularity coefficient of sand } \\
\text { Specific surface }\left(\mathrm{A}_{\text {BLAINE}}\right)\end{array}$ & 1.14 & 1.24 \\
\cline { 2 - 3 } $\mathrm{pH}$ & $120 \mathrm{~cm}^{2} / \mathrm{g}$ & $110 \mathrm{~cm}^{2} / \mathrm{g}$ \\
\cline { 2 - 3 } Density & 6.8 & 6.64 \\
\cline { 2 - 3 } Bulk density & $2.6 \mathrm{~g} / \mathrm{cm}^{3}$ & $2.6 \mathrm{~g} / \mathrm{cm}^{3}$ \\
\cline { 2 - 3 } & $1.6 \mathrm{~g} / \mathrm{cm}^{3}$ & $1.56 \mathrm{~g} / \mathrm{cm}^{3}$ \\
\hline
\end{tabular}

Table 1. Measurement data of the investigated sands. 


\section{Method/Measurements}

The ratio of cohesion and adhesion can be determined from the strength value results. In case of different surface qualities of samples with identical size distribution and binder quantity, equal bond strength developed. This means that the cohesion between sand grains was nearly the same. The difference between strength values may arise from the difference in the adhesion, which depends on the surface quality of the sand grains. Figure 3. shows the determination of the method for cohesion and adhesion.

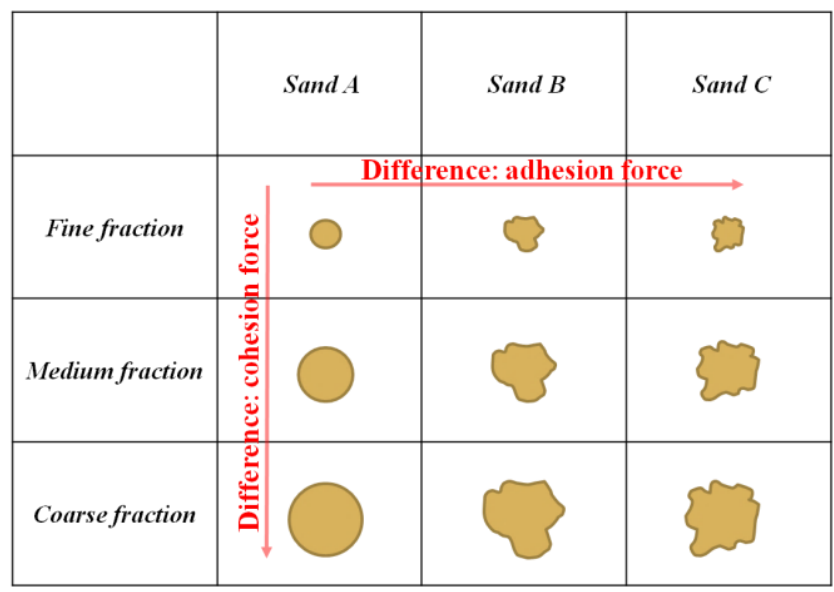

Figure 3. The method used for determining the cohesion and adhesion.

Different bentonite clay bonded sand mixtures were made from a different type of sands. Sands were added to mixtures with fractionation; the grain sizes were fine $(110-220 \mu \mathrm{m})$, medium $(220-300 \mu \mathrm{m})$ and coarse $(300-540 \mu \mathrm{m})$.

Test specimens of standard size ( $\varnothing 50 \mathrm{~mm}$ by $50 \mathrm{~mm}$ ) made from laboratory sand rammer of sand mix of $88 \%$ quartz sand, $8 \%$ bentonite with $4 \%$ moisture content after mulling with the Multiserw-Morek laboratory muller.

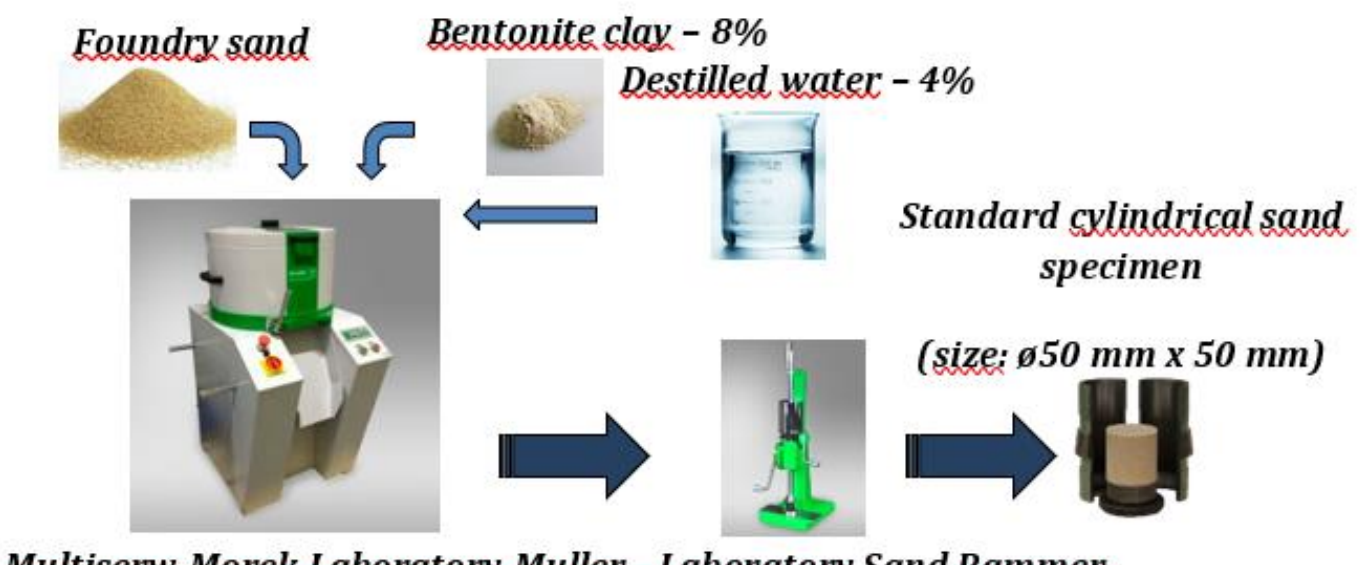

\section{Multiserw-Morek Laboratory Mullex Laboratory Sand Rammer}

Figure 4. Process of making test specimens 


\subsection{Permeability}

For the testing gas permeability required device is shown in Figure 5. where air with a defined pressure is pressed into and/or through the sample (Ø50 $\mathrm{mm}$ by $50 \mathrm{~mm}$ ). [6]

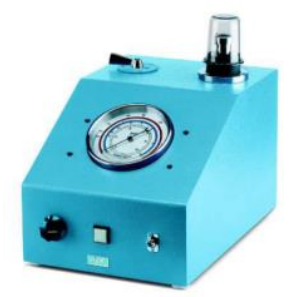

Figure 5. FED Electric Permeability Meter

\subsection{Compressive strength}

The standard test item (Ø50mm by $50 \mathrm{~mm}$; compressed with 3, 5, 7 and 9 rams), produced with a rammer is tested for compressive strength. The values were shown directly on the test device display in $\mathrm{N} / \mathrm{cm}^{2}$. [6]

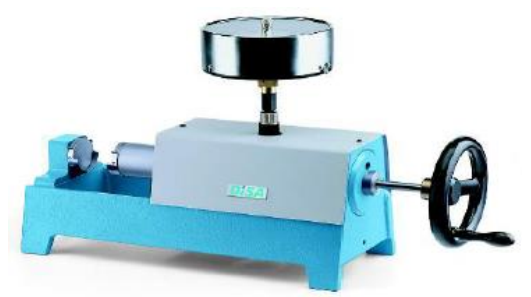

Figure 6. PFG Universal Strength Machine

\subsection{Wet tensile strength}

This strength can be measured by using the testing device for wet tensile strength shown in Figure 7. The sample shape is identical to the one for other strength tests, however, instead of a lid, a ring is put on the cylindrical tube. The compressed sample contained in the tube and ring a heated up $\left(300{ }^{\circ} \mathrm{C}\right)$ in the device so that a soaked area underneath the heated surface develops. It creates a zone of overwet sand which called condensation zone with reduced strength, a few millimetres below the surface. The ring is pulled off by a pneumatic device and the tensile strength of the soaked area is measured. Exposed to a tensile force the test specimen ruptures in this zone. The wet tensile strength in $\mathrm{N} / \mathrm{cm} 2$ is read from the maximum indicator pointer (up to $0.6 \mathrm{~N} / \mathrm{cm} 2$ ) and is a direct value for the evaluation of bentonite and moulding sands. [6]

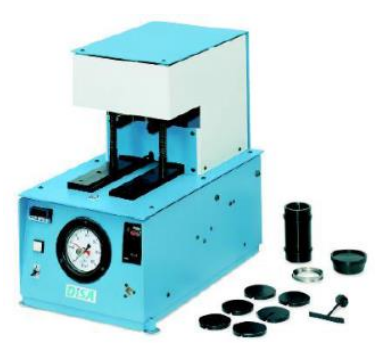

Figure 7. PNZ Wet Tensile Strength Testing Apparatus 


\section{Results}

The results of measurement tests are illustrated on Fig. 8-10. The illustrated measurement results come from the average of the 3 measurements.

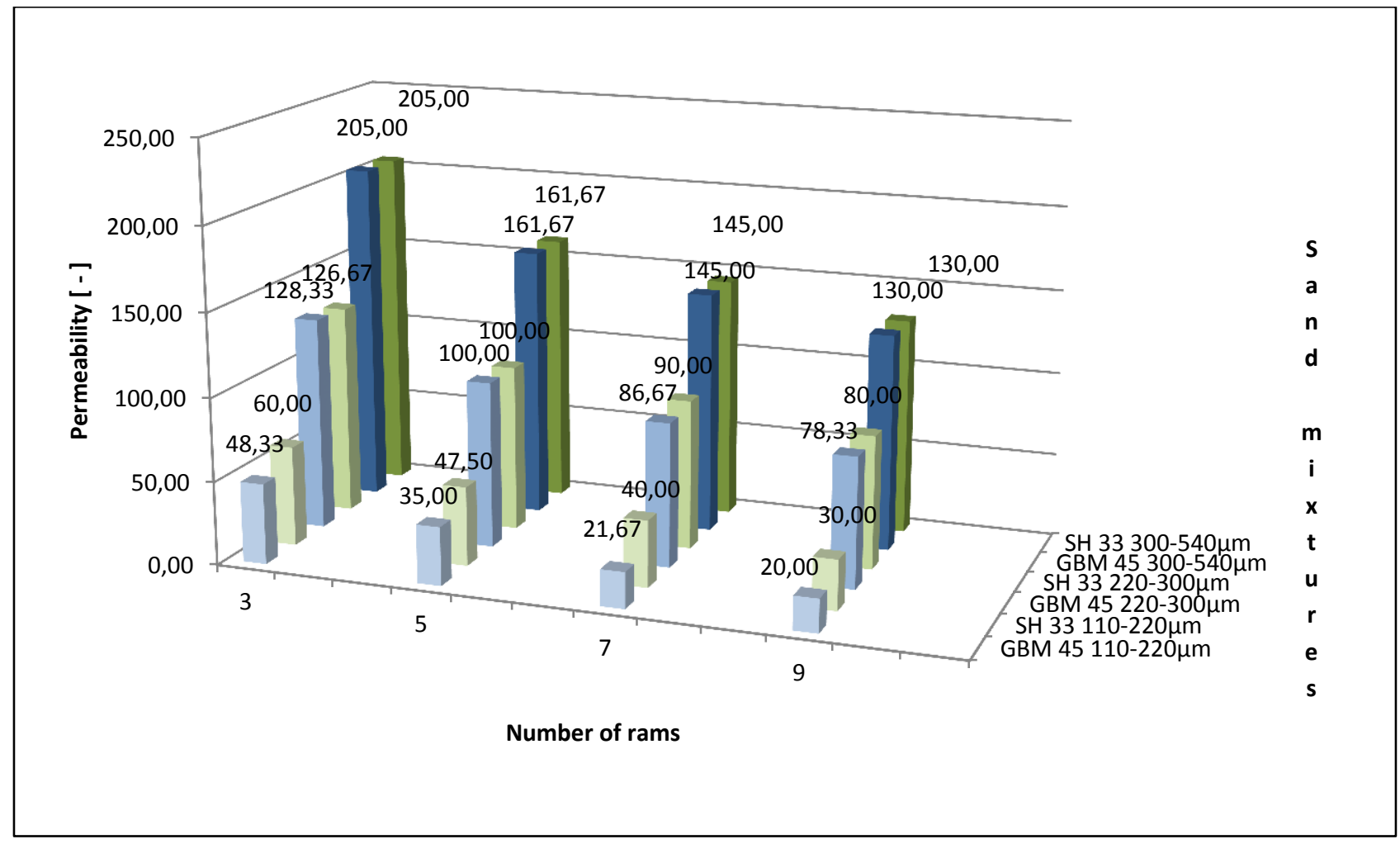

Figure 8. The permeability test results.

Figure 8. can be seen that the measured permeability of the specimens made of quartz sand type GBM 45 fine fraction is higher than that of the specimens made of SH 33 type sand, conversely the other two fractionations, where the measured permeability is nearly equal. In each case, the number of rams increase in raise gives a lower permeability. 


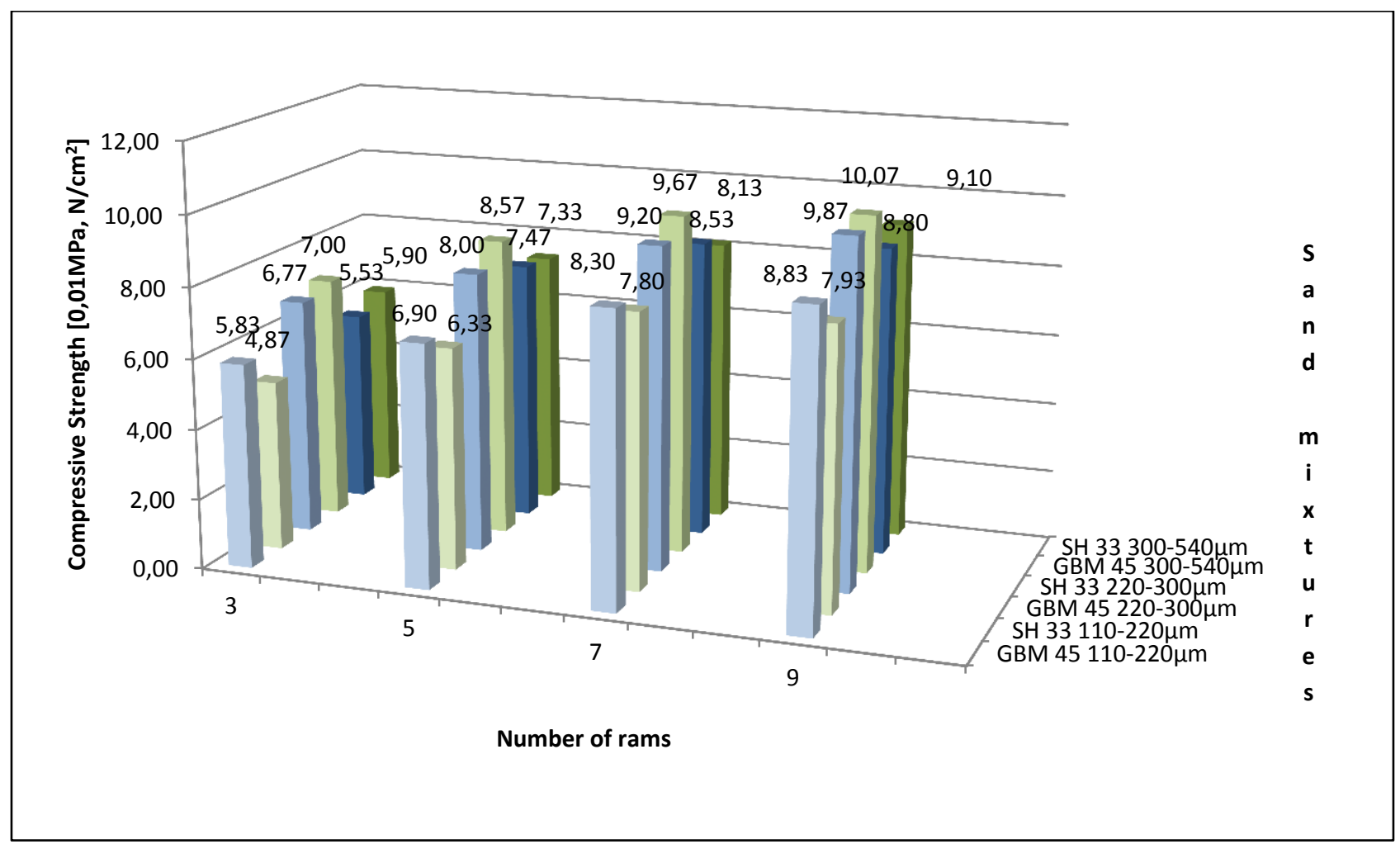

Figure 9. The compressive strength test results.

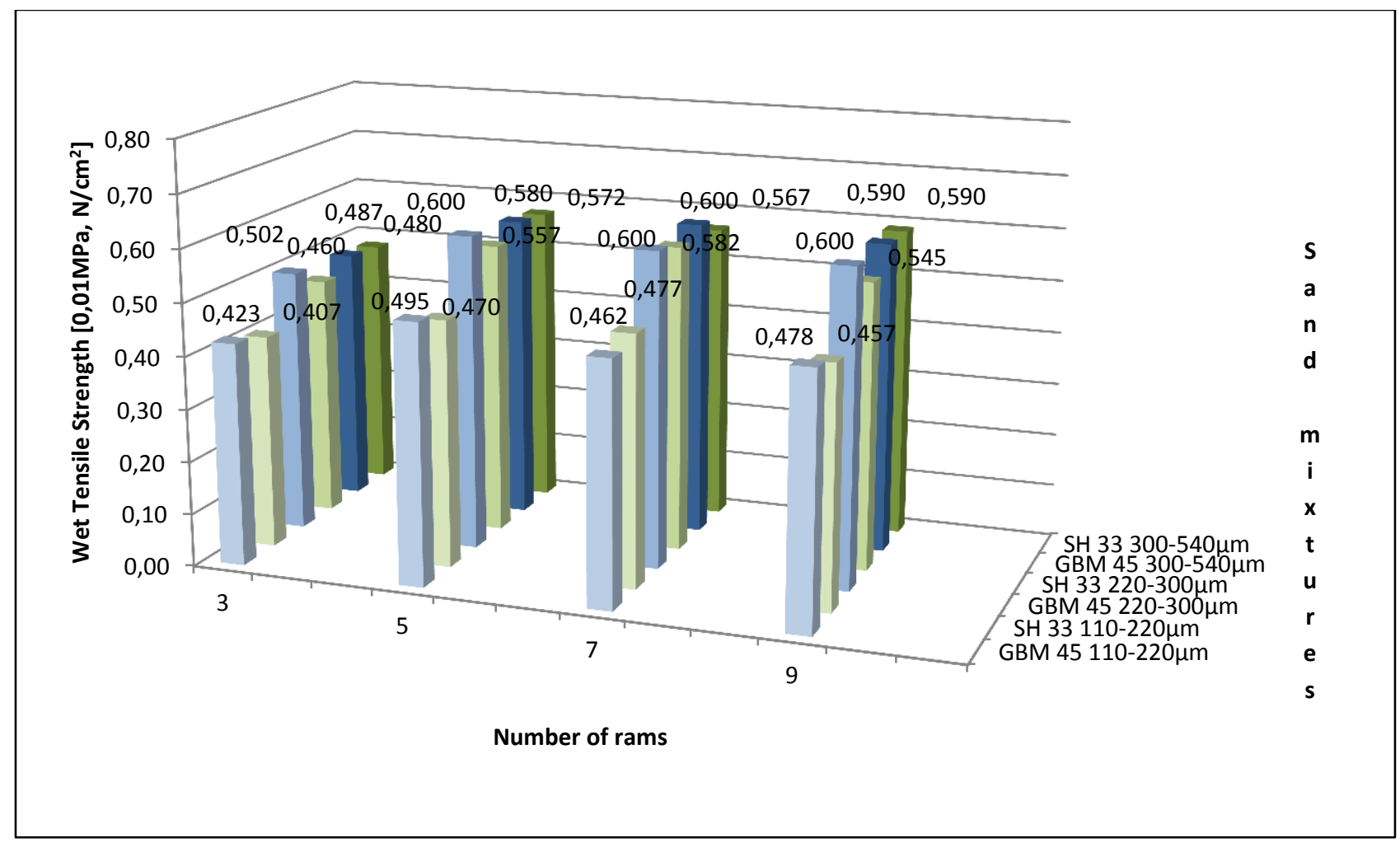

Figure 10. The wet tensile strength test results.

Based on the results of the compressive strength and also the wet tensile strength (the Fig. 9-10), it can be seen that the measured strength of the specimens made of quartz sand type GBM 45 (even in 
case of different fractions) in most cases is higher than that of the specimens made of SH 33 type sand. Compressive strength values of the specimens made of medium fraction sands are the highest as compared to coarse or fine fraction - in case of both types (GBM 45 and SH 33) of sands. At the same time, wet tensile strength values of the specimens made of coarse fraction sands are mostly higher, and the specimens made of fine fraction sands are smaller - in case of both types (GBM 45 and SH 33) of sand.

\section{Conclusion}

A comparative measurement of two quartz sand - GBM 45 and SH 33 (with different fractionations fine, medium and coarse) with different surface quality was carried out. To ensure the number of bonding bridges and the same connecting points of the sand grains the fractionation was needed. Greensand mixtures were prepared to measure their permeability, compressive strength and wet tensile strength.

It is possible to determine the ratio of cohesive and adhesive forces within the strength values based on the test results carried out. The difference in the strength values may be due to the different adhesion energy, which depends on the surface quality of the sand grains.

The results of the investigation show that foundry sands with different micro surfaces can have different binding strength values. With the help of the fractionation method of the sand grains, the cohesion and adhesion ratio can be determined from the measured strength values.

\section{References}

[1] K. Löchte, Working with the Cold Box Process in the Coremaking Department of a Foundry, Internet, 1998.

[2] V. Bechný, Zukünftige Herausforderungen an Gießereisande, Giesserei-Rundschau, 2012 3/4,,81-83.

[3] F. Iden, W. Tilch, H. J. Wojtas, Die Haftungsmechanismen von Cold-Box-Bindemitteln auf der Formstoffoberfläche, Giesserei, 2011/5., 24-36.

[4] F. Iden, U. Pohlmann, W. Tilch, H. J. Wojtas, Strukturen von Cold-Box-Bindersystemen und die Möglichkeit ihrer Veränderung, Giesserei Rundschau, 2011 1⁄2., 3-8.

[5] http://giba.at/pdf/giba-de.pdf

[6] https://www.giessereilexikon.com 